\title{
ON THE AREA OF HARMONIC SURFACES
}

\author{
MICHAEL BEESON
}

\begin{abstract}
We prove that if the sequence $S_{n}$ of harmonic surfaces converges to the harmonic surface $S$, and if the boundary of $S_{n}$ is a rectifiable Jordan curve $C_{n}$, whose length is uniformly bounded by $L$, then the area $A\left(S_{n}\right)$ converges to $A(S)$. This solves an old problem, several special cases of which have been solved in the literature.
\end{abstract}

The question addressed in this paper is the following: suppose a sequence $S_{n}$ of surfaces converges to a surface $S$. Then does the area $A\left(S_{n}\right)$ converge to the area $A(S)$ ? Of course it does, if the surfaces are smooth and convergence is measured in a strong norm. On the other hand, here we are concerned with sup-norm convergence; in that case, the surfaces $S_{n}$ may have many small wrinkles which cause $\lim \inf A\left(S_{n}\right)$ to be larger than $A(S)$. Hence some additional hypothesis is necessary. A natural hypothesis is to require the surfaces $S_{n}$ and $S$ to be harmonic surfaces, i.e. to be given by harmonic functions $X(u, v), Y(u, v)$, and $Z(u, v)$. Even this hypothesis, which certainly eliminates "wrinkles", is not sufficient. Let $S_{n}$ be bounded by a curve $C_{n}$, where $C_{n}$ is formed by putting many small loops in a spiral around the unit circle, like an electric filament. If we put, say, $n^{2}$ loops of "radius" about $1 / n$, one sees intuitively that the area of $S_{n}$ will contain a term like $n^{2} \Sigma \pi / n^{2}$, which will not vanish in the limit as $S_{n}$ converges to the unit disk. (A less intuitive example is made rigorous in [M-T].) In this example, however, the length of the boundary curves $C_{n}$ tends to infinity. The natural conjecture was then that if the lengths $L\left(C_{n}\right)$ are uniformly bounded, the $S_{n}$ are harmonic and converge to a harmonic $S$, then $A\left(S_{n}\right)$ converges to $A(S)$. The purpose of this note is to prove this conjecture.

The history of the problem is as follows: Morse and Tompkins proved a similar theorem, but under the additional assumption that the lengths of the boundaries $C_{n}$ converge to the length of $C$. They explicitly stated the conjecture solved in the present paper, perhaps for the first time in print; that was 1941. In 1950, Baiada was able to weaken the hypothesis somewhat, but the conjecture remained unsolved. An interesting special case, in which the harmonic surfaces are minimal surfaces (i.e. provide a conformal mapping, except at branch points), was treated by Shiffman [S, p. 104] and by Courant [C, p. 131]; Shiffman's paper was published in 1942.

There may well be other ways to rule out "wrinkles" than by requiring

Received by the editors July 7, 1977.

AMS (MOS) subject classifications (1970). Primary 53A05.

C American Mathematical Society 1978 
harmonicity. For instance, under the assumption that the $S_{n}$ are surfaces of constant mean curvature (in the sense that $\Delta S=H S_{u} \wedge S_{v}$ together with a conformality condition), the problem was solved under the assumption of uniformly bounded boundary lengths by Heinz [H, p. 262]. This result includes the minimal surface case $(H=0)$, but neither implies nor is implied by the result of the present note.

The present author discovered the problem from a quite different approach, and only later learned of its long history. He was studying Plateau's problem from a constructive viewpoint, and came to the point of defining the area $A(S)$ for harmonic surfaces $S$. The area is given by a certain integral

$$
A(S)=\iint W d u d v=\int_{0}^{2 \pi} \int_{0}^{1} W r d r d \theta=\lim _{r \rightarrow 1} \int_{0}^{2 \pi} \int_{0}^{r} W r d r d \theta .
$$

Constructively speaking, we must show how to compute the area. That means, given $\varepsilon>0$, we must be able to know how close to 1 we must take $r$ so that $\int_{0}^{2 \pi} \int_{0}^{r} W d r d \theta$ is within $\varepsilon$ of $A(S)$. Of course, in general the area may be infinite; but we want to prove that if the boundary is rectifiable, then the area is well defined. This means we must estimate the area $A_{r 1}=\int_{0}^{2 \pi} \int_{r}^{1} W r d r d \theta$ in terms of a bound $L$ on the length of the boundary. (Constructively, it is weaker to have a bound $L$ on the length than to be able to compute the length; and the weaker hypothesis is more useful.) Such an estimate thus appears as a basic necessity for the constructive theory of harmonic surfaces.

It so happens that an estimate of the quantity $A_{r 1}$ is exactly what is needed to prove the theorem on continuity of area with which we are concerned. Previous work on the problem has not led to an estimate only in terms of the boundary length; once we have that, the theorem follows easily, as we show here. This is not accidental; there is a fundamental connection between constructivity and continuity, which may be stated roughly as follows: if a problem can be constructively solved, then the solution depends continuously on parameters. The present theorem illustrates this principle, where the problem is, find the area of a harmonic surface whose boundary has length $\leqslant$ $L$. Further discussion may be found in [B1] and [B2].

We now proceed with the details. For simplicity, we consider only surfaces of the topological type of the disk. Thus a surface is a map from the open disk $P$ to $R^{3}$. We consider only harmonic surfaces, i.e. we require $\Delta \vec{x}=0$ in $P$. We allow "branch points" where $x_{u}=x_{v}=0$; these must be isolated, since with $z=u+i v$, they are the simultaneous zeroes of the three analytic functions $d x_{i} / d z$. We consider only surfaces which extend continuously to the closed disk $\bar{P}$ and when restricted to the boundary, provide a homeomorphism of the unit circle into $R^{3}$. If this homeomorphism is a reparametrization of the Jordan curve $\Gamma$, we say $x$ is bounded by $\Gamma$. We consider only surfaces bounded by a rectifiable Jordan curve.

A modulus of continuity for $\vec{x}$ is a function $\delta(\varepsilon)$ such that $|z-w|<\delta(\varepsilon)$ $\rightarrow|x(z)-x(w)|<\varepsilon$ for all $z, w \in \bar{P}$. By $W$ we mean the element of area, so 
that the area is given by $\iint_{P} W d u d v$. (We have $W^{2}=\vec{x}_{u}^{2} \cdot \vec{x}_{v}^{2}-\left(\vec{x}_{u} \cdot \vec{x}_{v}\right)^{2}$.) By $A_{r s}(\vec{x})$, or just $A_{r s}$ if $\vec{x}$ is clear in context, we mean the area of the image under $x$ of the annulus $r<|z|<s$. By $A_{r}$ we mean $A_{0 r}$.

REMARK. For our constructive study [B1], we need a constructive proof of Theorem I below; we take this opportunity to note that we have paid attention to the constructivity of the arguments.

LEMMA 1. $A[\vec{x}]<\frac{1}{2} L \operatorname{osc}(\vec{x})$, where $x$ is any harmonic surface whose boundary has length $\leqslant L ; \operatorname{osc}(\vec{x})$ is a bound for the difference of any two values of $x$.

Proof [N, §324]. The proof there has to be followed back a section or two to be sure that it works under the hypothesis that $\vec{x}$ is only harmonic, not necessarily minimal. It is constructive, as long as the domain in which $x$ is parametrized is (constructively) conformally equivalent to a disk.

LEMMA 2. Let $\vec{x}$ be a harmonic surface (in the unit disc) whose boundary is a curve of length $<L$. Let $N$ be a natural number and let $\varepsilon>0$. Then we can find an angle $\theta$ such that, with $\theta_{i}=\theta+2 \pi i / N$, we have for $r \geqslant \frac{4}{5}$,

$$
h(\theta)=\sum_{i=0}^{N-1} \int_{r}^{1}\left|x_{r}\left(r, \theta_{i}\right)\right| d r<\frac{N}{2 \pi}\left(4 L \sqrt{1-r}+\frac{\varepsilon}{N}\right) .
$$

REMARK. Nonconstructively, $\varepsilon$ would be superfluous, but the lemma would be no simpler.

ProOF.

$$
\vec{x}_{r}(r, \theta)=\int_{0}^{2 \pi} \frac{\sin (\xi-\theta)}{1-2 r \cos (\xi-\theta)+r^{2}}|d \vec{x}(\xi)|
$$

(see [C, p. 135]).

Hence

$$
B=\int_{0}^{2 \pi} \int_{r}^{1}\left|x_{r}(r, \theta)\right| d r d \theta \leqslant \int_{0}^{2 \pi} \int_{r}^{1} \int_{0}^{2 \pi} \frac{|\sin (\xi-\theta)|}{1-2 r \cdot \cos (\xi-\theta)+r^{2}}|d \vec{x}(\xi)| .
$$

Interchanging the order of integration, performing the $\theta$-integration first, and using the fact that

$$
\int_{0}^{2 \pi} \frac{|\sin \xi|}{1-2 r \cos \xi+r^{2}} d \xi=\frac{2}{r} \log \left(\frac{1+r}{1-r}\right)
$$

this yields

$$
B \leqslant \int_{0}^{2 \pi} \int_{r}^{1} \frac{2}{r} \log \left(\frac{1+r}{1-r}\right) d r|d \vec{x}(\xi)| .
$$

We also can do the $r$-integration explicitly. Put $\rho=1-r ; v=2 / \rho$. Then

$$
\int_{r}^{1} \frac{2}{r} \log \left(\frac{1+r}{1-r}\right) \leqslant \int_{0}^{\rho} 4 \log \frac{2}{\rho} d \rho=\int_{2 / \rho}^{\infty} 8 \frac{\log v}{v^{2}} d v
$$


since $r \geqslant \frac{4}{5}$, we have $\log v \leqslant \sqrt{v}$, so the integral is

$$
\leqslant \int_{2 / \rho}^{\infty} 8 v^{-3 / 2} d v \leqslant 4 \sqrt{\rho}
$$

Thus

$$
B \leqslant \int_{0}^{2 \pi} 4 \sqrt{1-r}|d \vec{x}(\xi)| \leqslant 4 L \sqrt{1-r} .
$$

Since $B=\int_{0}^{2 \pi / N} h(\theta) d \theta$, we can apply the (constructive) mean value theorem to find $\theta$ such that $h(\theta) \leqslant(N / 2 \pi)(4 L \sqrt{1-r}+\varepsilon / N)$, proving Lemma 5.2.

THEOREM I. Let $x$ be a harmonic surface whose boundary $\Gamma$ has length $L$. Then the area $A(x)$ is constructively well defined. That is, given $\varepsilon>0$, we can explicitly produce $\delta>0$ (in terms of $L$ and a modulus of continuity for $x$ only) such that $|1-r|<\delta$ implies $A_{r 1}<\varepsilon$.

THEOREM II. Let $S_{n}$ be a sequence of harmonic surfaces converging to the harmonic surface $S$, whose boundary curves $C_{n}$ all have length $\leqslant L$. Then the area $A\left(S_{n}\right)$ converges to $A(S)$.

Proof. Theorem II follows from Theorem I exactly as in [N, §327], nonconstructively. A constructive proof is given in [B1], using some metamathematical principles; this proof provides (in principle) a way of getting from $\varepsilon$ an explicit $N(\varepsilon)$ such that $k \geqslant N(\varepsilon)$ implies $\left|A\left(S_{k}\right)-A(S)\right|<\varepsilon$. Theorem $I$ is proved by arguments similar to, but slightly different from, [N, §326]; we give this proof. If $\varepsilon>0$ is given, choose $N$ so large that $1 / N<\delta(\varepsilon / 2 L)$, the modulus of continuity for $\vec{x}$. Then choose $\theta$ as in Lemma 2, and consider the $N$ "boxes" into which the lines $\phi=\theta_{i}$ divide the annulus between $r$ and 1. Choose $r$ so near 1 that $\sqrt{1-r} \leqslant 1 / N$; then also $1-r \leqslant 1 / N$, so the oscillation of $\vec{x}$ on each "box" is less than $\varepsilon / 2 L$. By Lemma 1, the area $A_{i}$ of the image of the $i$ th "box" is less than $\varepsilon P_{i} / 4 L$, where $P_{i}$ is the perimeter of the $i$ th box. Hence the total area of the image of the annulus $A_{r 1}$ is less than $(\varepsilon / 4 L) \sum P_{i}$; but $\sum P_{i}$ is just $L+L(r)+$ $2 \sum \int_{r}^{1}\left|x_{r}\left(\theta_{i}\right)\right| d r \leqslant L+L(r)+h(\theta)$, where $h(\theta)$ is as in Lemma 2, and $L(r)$ is the length $\int_{0}^{2 \pi}\left|x_{\theta}(r, \phi)\right| d \phi$. It is easy to show $L(r) \leqslant L$ (see [C, p. 127], for instance) and applying Lemma 2 , we have

$$
A_{r 1} \leqslant \frac{\varepsilon}{4 L}\left(2 L+\frac{N}{\pi}\left(4 L \sqrt{1-r}+\frac{\varepsilon}{N}\right)\right)
$$

since $\sqrt{1-r} \leqslant 1 / N$, we have

$$
A_{r 1} \leqslant(\varepsilon / 4 L)(7 L / 2+\varepsilon / 2 \pi) \leqslant \varepsilon .
$$

This completes the proof.

\section{REFERENCES}

[Ba] E. Baiada, L'area delle superficie armoniche quale funzione delle rappresentazioni del contorno, Riv. Mat. Univ. Parma 2 (1951), 315-330.

[B1] M. Beeson, Plateau's problem and constructive mathematics (to appear).

[B2] __ Principles of continuous choice and continuity of functions in formal systems for 
constructive mathematics, Ann. Math. Logic (to appear).

[C] R. Courant, Dirichlet's principle, conformal mapping, and minimal surfaces, Interscience, New York, 1950.

[H] E. Heinz, Unstable surfaces of constant mean curoature, Arch. Rational Mech. Anal. 38 (1970), 256-267.

[M-T] M. Morse and C. Tompkins, The continuity of the area of harmonic surfaces as a function of the boundary representation, Amer. J. Math. 63 (1941), 825-838.

[N] J. C. C. Nitsche, Vorlesungen über Minimalflachen, Springer-Verlag, Berlin and New York, 1975.

[S] M. Shiffman, Unstable minimal surfaces with any rectifiable boundary, Proc. Nat. Acad. Sci. USA 28 (1942), 103-108.

Current address: Department of Mathematics, Stanford University, Stanford, California 94305 